\title{
Hybrid immunity: the immune response of COVID-19 survivors to vaccination
}

\author{
Marlon Gancino ${ }^{1}$, Nelson Santiago Vispo ${ }^{2}$
}

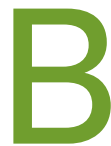

$y$ the time writing this editorial, around nineteen months (Dec 2019) has passed since the first report of a Severe Acute Respiratory Syndrome Coronavirus 2 (SARS-CoV-2) outbreak in individuals of Wuhan, China $^{1}$. Also, it has been more than sixteen months (Mar 2020) since the declaration of the Coronavirus disease 19 (COVID-19) pandemic by the World Health Organization (WHO) ${ }^{1}$. The evolution and rapid scattering of this novel coronavirus have trapped in an unprecedented global public health crisis, which, so far, has reached 196,553,009 people around the globe, provoking 4,200,412 deaths (31st July 2021)1.

The global endeavor of defeating contagion has achieved groundbreaking events such as the fastest development and massive application of effective vaccines in history ${ }^{2}$. Currently, +500 SARS-CoV-2 vaccines are being developed over the full spectrum of platforms (i.e., non-viral vector, viral vector, inactivated and live-attenuated virus, and recombinant vaccines) some of which are already pre-clinical and clinical studies ${ }^{2,3}$ Remarkably, ten vaccines are already available on the market ${ }^{4}$, from which, by the end of July 2021, +3800 million doses have been administered ${ }^{1}$. Complemented by non-pharmaceutical strategies (e.g., restrictions on international mobility), inducing protective immunity to SARS-CoV-2 massively until attaining herd immunity appears to be the definitive solution to overcome this pandemic ${ }^{5}$.

We are reaching milestones daily, so some exciting questions have been raised about immunological memory - the fundamental of protective immunology. This response coordinated by a synchronized orchestra of antibodies, memory B cells, memory CD4+ $T$ cells, and memory CD8+ $T$ cells remains poorly understood ${ }^{6}$. Either infection (natural immunity) or vaccination (vaccine-induced immunity) can trigger immunological memory against SARS-CoV-2, and all nuances in such complexity may provoke different biological outcomes? Diverse investigations have concluded that either path to immunity can confer specific protection to COVID-198-11. Moreover, they have discussed the question: what does it occur when these immunities are overlapped? Acquiring knowledge on the immune response dynamics that convalescent individuals who are subsequently vaccinated develop is essential. The deep understanding of this "hybrid immunity" will allow the design of appropriate vaccination regimes for the vast population of previously infected individuals ${ }^{12}$.

Observations on the response kinetics of immune memory from naturally immunized cohorts have found that 95\% of subjects can develop a stable immunity against reinfection, which may last moderately declining over a year after symptoms onset ${ }^{6,13}$. Relevantly, such protective immunity suffers a considerable weakening in the face of current circulating SARS-CoV-2 variants of concern (VOCs) - i.e., B.1.1.7 (Alpha), B.1.351 (Beta), P.1 (Gamma), and B.1.617.2 (Delta) - when compared with the wild-type Wuhan-Hu-1 variant ${ }^{4,14-16}$. As identified by previous works, multiple spike (S) protein mutations in VOCs explain their ability to escape neutralization antibody activity partially, making the acquired natural protective immunology less effective ${ }^{7,12}$.

Although to a milder degree, reduction to VOCs neutralization has also been seen in vaccine immunized people ${ }^{16}$ Currently, four main types of vaccines govern the market: mRNA-lipid nanoparticle-based non-viral vectors (BNT162b2 by Pfizer-BioNTech, and mRNA-1273 by Moderna), adenovirus-based viral vectors (ChAdOxI-nCoV-19 by AstraZeneca, Ad26.COV2.S by Johnson \& Johnson, Ad5-nCoV by CanSino Biologics, and Sputnik V by Gamaleya), inactivated virus (CoronaVac by Sinovac, BBV152 by Bharat Biotech, WIBP-CorV by Sinopharm (Wuhan), and BBIBP-CorV by Sinopharm (Beijing)), and recombinant protein subunits (NVX-CoV2373 by Novavax) $)^{3,4}$. Phase 3 clinical trials showed good overall vaccine efficacies that ranged from -95\% (BNT162b2) to $>50 \%$ (CoronaVac) at preventing COVID-19 illness, commonly after a two-dose prime-boost regime ${ }^{17}$. In such a sense, it has been observed that vaccination protection effectiveness slightly reduces against VOCs, Delta variant being associated with the highest transmissibility and ability to avoid antibody neutralization ${ }^{16}$. However, although less potent to VOCs, preliminary research suggests that vaccines probably remain efficacious ${ }^{8,11,16,18}$. Now, it is statistically modelized that vaccine-induced neutralization activity would decay in a long non-linearly fashion, causing the vaccines with initial efficacy of $95 \%$ to preserve $77 \%$ efficacy in a 250 -day span ${ }^{19}$.

Identifying the differences between natural and vaccine-induced immunities directs us to the proper interrogation: what is there at their interface? As mentioned earlier, numerous research groups have addressed this question, and their results suggest that hybrid immunity has a more robust protective performance than either immunity. In a synergistic character, researchers have seen that previously infected vaccinees mount cross-variant neutralization reactogenicity to the first vaccine dose, which equals or surpasses the observed in naïve individuals after the second vaccine shot $t^{5,7,8,11,16,20,21}$.

Reports in this regard informed the occurrence of anamnestic reactions in diverse cohorts of previously infected vaccinees after receiving the first vaccine dose of either BNT162b2, mRNA-1273, or ChAdOx1-nCoV-19 with no apparent improvement after the second dose $e^{7-9,11,17,21}$. According to Goel et al. and Kramer et al., as anticipated, only COVID-19 survivors present detectable levels of immunoglobulin $\mathrm{G}(\mathrm{lg} G)$ antibodies specialized to recognize the full-length SARS-CoV-2 S protein (anti-S IgG) or the SARS-CoV-2 spike receptor-binding domain (RBD) (anti-RBD lgG) - antibodies associated with SARSCoV-2 neutralization responses ${ }^{13}$ - before vaccination ${ }^{17,22}$. As observed, titers of anti-S IgG and anti-RBD IgG are higher in individuals with a history of SARS-CoV-2 infection pre- and post-vaccination (compared with naïve subjects at the exact sampling times) ${ }^{17,20,23}$. The first vaccine dose induces a variable and inadequate response of neutralizing antibodies generation in SARS-CoV-2-unexposed individuals, which boosts the second vaccination ${ }^{24}$. Contrary, the first vaccine dose triggers 


\section{Synergistic Immune Response of COVID-19 Convalescent Individuals After Vaccination}

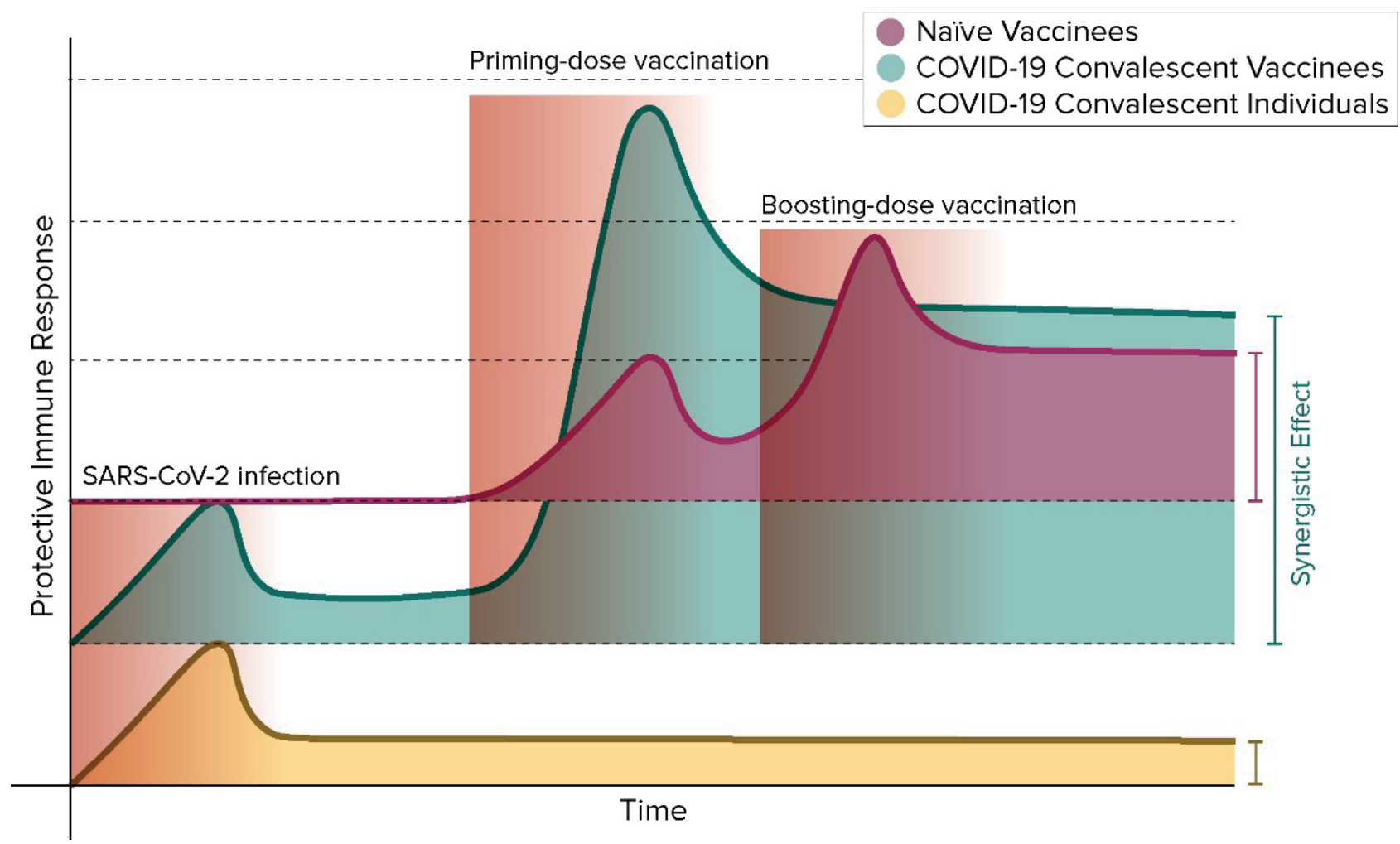

a uniform and rapid amplification of anti-S IgGs and anti-RBD IgGs titers in SARS-CoV-2 experienced individuals ${ }^{10,11}$. While naïve and COVID-19 asymptomatic vaccinees' antibody titers are significantly boosted after the second vaccine dose ${ }^{21}$, no significant improvement has been detected in participants with preexisting immunity after the boosting dose, suggesting a possible plateau in the neutralization antibody activity 9,11,20.

Interestingly, as informed by Ebinger et al., neutralizing antibodies levels in naïve individuals who received the first vaccine dose were slightly higher when contrasted with the baseline state of previously infected individuals ${ }^{23}$. In their research, neutralizing antibody levels were statistically similar in both groups, only if compared to the group of previously infected individuals after the first dose with the group of naïve individuals after completing a two-dose vaccination regime ${ }^{23}$. Also, higher frequencies of vaccine-related systemic side effects (e.g., fever, headache, muscle pain, and fatigue) have been associated with COVID-19 convalescent individuals and high IgG responses ${ }^{9}$.

As a complement, the humoral immune response of SARS-CoV-2-exposed individuals is boosted above the protective threshold after the first vaccination dose, even against $\mathrm{VOCs}^{8,16}$. Planas et al. noticed that sera from previously infected one-dose vaccinees displayed a significant increase in neutralizing antibody titers against Alpha, Beta, and Delta SARSCoV-2 variants, in contrast with non-vaccinated convalescent people ${ }^{16}$. On the contrary, such cross-variant neutralization activity was observed in naïve individuals just days after the second vaccine immunization. Therefore, these emerging data highly advise that SARS-CoV-2 experienced individuals subsequently vaccinated may develop a broad protective immunity against all spreading VOCs $\mathrm{S}^{7,16}$.
Every compartment of the adaptative immune systems is probably involved in prompting this non-anticipated hybrid immunity behaviour ${ }^{7}$. Broadly, as part of the immune response against infection, long-live memory B cells by a previous induction of T cells (specifically, T follicular helper cells) start compiling mutations in immunoglobulin variable gene sequences (somatic hypermutations (SHM)) in favor of a future generation of high-affinity antibodies ${ }^{13}$. Thus, evolved memory B cells can be recruited post-vaccination, which will differentiate into plasma cells producing highly potent neutralizing antibodies ${ }^{13}$. It has been observed that memory B cells continue accumulating somatic mutations over 12 months in germinal centers in individuals who have recovered from COVID-1913,25. Those mutations accumulated long after infection are, indeed, though accountable for the high potency in serum neutralization activity of the convalescent vaccinee cohorts against VOCs ${ }^{12}$. Although the study performed by Goel et al. it was not found any change in the levels of SHMs in memory B cell clones from convalescent individuals as a response to vaccination ${ }^{17}$, naïve vaccinees also would enter into this memory B cell maturation after antigen exposure (vaccination), if analyzed after long spans $^{12}$.

The prime-boost regime strategy chose for most vaccines relies on mounting protective responses through memory recall to antigen reexposure ${ }^{7}$. The natural SARS-CoV-2 infection primes convalescent populations. This primary encounter with the virus promotes the previously infected individuals to develop a $T$ cell memory response to a vast epitope repertoire of the 25 SARS-CoV-2 proteins (S and non-S viral proteins), which according to several studies, are maintained in VOCs ${ }^{7,15,20}$. Most commercial vaccines induce a protective response using only the SARS-CoV-2 S-protein,11. Accordingly, vaccine priming 
activates a $T$ cell memory to a restricted number of epitopes in naïve individuals (compared to SARS-CoV-2-exposed individuals) ${ }^{7}$. Therefore, the reactivation of broad infection-primed $T$ cell memory response and the recruitment of long-live mutated memory B cell after first vaccine dose in SARS-CoV-2-exposed individuals probably play a role in the astounding response kinetics of hybrid immunity ${ }^{20}$.

The boosting vaccine dose produced in $\mathrm{B}$ and $\mathrm{T}$ cell kinetics is remarkably similar to the one observed in the serological analysis. That is, boosting effect of the second vaccine dose was only observed in naïve individual ${ }^{8,11,17,20}$. Humoral and cellular immunity of convalescent vaccinees may reach an activity plateau after the first vaccine dose $^{20}$. In this sense, the study of Lozano-Ojalvo et al. reported a reduction in $T$ cell responses in COVID-19 recovered individuals after the second vaccine dose ${ }^{20}$. Taken together, those results reveal that convalescing individuals may request an alternative vaccination regime different that the current prime-boost one developed in naïve individuals.

Global health systems are still battling a colossal challenge aggravated by the shortage in vaccine supply and the occurrence and dissemination of VOCs ${ }^{5,21}$. Vaccine policies are being discussed worldwide, proposing several strategies to address herd immunity to protect their populations ${ }^{15,24}$. A clear example was seen in the United Kingdom when authorities approved extending the time interval between vaccine dosages to apply the first vaccine immunization to the most significant number of citizens ${ }^{8}$. However, as above expose, one dose in naïve individuals may not be enough to protect them from VOCs, such as the Delta variant ${ }^{16}$.

Hybrid immunity can become a turning-point opportunity to defeat the infection in context with the current pandemic scenario. Adopting policies aligned with the data mentioned above may be particularly beneficial for regions like Latin America. While this region shares only $-5 \%$ of the world population, it accumulates $+16 \%$ of the -200 million COVID-19 total cases, so far reported ${ }^{1}$. Acknowledging the reduced diagnostic capabilities of these countries, this entire area is probably one of the most affected globally, leading to one of the more numerous populations of convalescent people. From both an economic and pharmacological perspective, applying a one-dose regime of pertinent vaccines to convalescent individuals is sustainable ${ }^{5}$. As COVID-19 survivors may need just one vaccination to achieve high levels of protective immunity, massive antibody screening for SARS-CoV-2 spike antibodies could help prioritize and free up doses, optimize vaccine supply efficiency, and surpass problems linked to the current vaccine manufacturing bottleneck ${ }^{5,25,26}$.

Finally, as all cited reports were performed considering vaccines developed over only mRNA-lipid nanoparticle-based non-viral vectors and adenovirus-based viral vectors, it is vital to extend the research to the rest of marketed vaccines and figure out if the same "hybrid immunity" principles apply to them.

\section{Bibliographic references}

1. World Health Organization. Coronavirus (COVID-19) Dashboard. World Health Organization (2021). Available at: https://covid19. who.int/. (Accessed: 31st July 2021)

2. Hrkach, J. \& Langer, R. From micro to nano: evolution and impact of drug delivery in treating disease. Drug Deliv. Transl. Res. 10, 567-570 (2020).
3. Li, Q., Wang, J., Tang, Y. \& Lu, H. Next-generation COVID-19 vaccines: Opportunities for vaccine development and challenges in tackling COVID-19. Drug Discov. Ther. 15, 2021.01058 (2021).

4. Focosi, D., Tuccori, M., Baj, A. \& Maggi, F. SARS-CoV-2 Variants: A Synopsis of In Vitro Efficacy Data of Convalescent Plasma, Currently Marketed Vaccines, and Monoclonal Antibodies. Viruses 13, 1211 (2021).

5. Focosi, D., Baj, A. \& Maggi, F. Is a single COVID-19 vaccine dose enough in convalescents? Hum. Vaccines Immunother. 00, 1-3 (2021).

6. Dan, J. M. et al. Immunological memory to SARS-CoV-2 assessed for up to 8 months after infection. Science 371, eabf4063 (2021).

7. Crotty, S. Hybrid immunity. Science 372, 1392-1393 (2021).

8. Reynolds, C. J. et al. Prior SARS-CoV-2 infection rescues B and T cell responses to variants after first vaccine dose. Science 372 , 1418-1423 (2021).

9. Sasikala, M. et al. Immunological memory and neutralizing activity to a single dose of COVID-19 vaccine in previously infected individuals. Int. J. Infect. Dis. 108, 183-186 (2021).

10.Saadat, S. et al. Binding and Neutralization Antibody Titers After a Single Vaccine Dose in Health Care Workers Previously Infected With SARS-CoV-2. JAMA 325, 1467 (2021).

11. Stamatatos, L. et al. mRNA vaccination boosts cross-variant neutralizing antibodies elicited by SARS-CoV-2 infection. Science 372, 1413-1418 (2021).

12. Purushotham, J. N., van Doremalen, N. \& Munster, V. J. SARSCoV-2 vaccines: anamnestic response in previously infected recipients. Cell Res. 2-3 (2021). doi:10.1038/s41422-021-00516-7

13. Wang, Z. et al. Naturally enhanced neutralizing breadth against SARS-CoV-2 one year after infection. Nature (2021). doi:10.1038/ s41586-021-03696-9

14. Moyo-Gwete, T. et al. Cross-Reactive Neutralizing Antibody Responses Elicited by SARS-CoV-2 501Y.V2 (B.1.351). N. Engl. J. Med. 384, 2161-2163 (2021).

15. Noh, J. Y., Jeong, H. W. \& Shin, E. C. SARS-CoV-2 mutations, vaccines, and immunity: implication of variants of concern. Signal Transduct. Target. Ther. 6, 3-4 (2021).

16. Planas, D. et al. Reduced sensitivity of SARS-CoV-2 variant Delta to antibody neutralization. Nature (2021). doi:10.1038/s41586021-03777-9

17. Goel, R. R. et al. Distinct antibody and memory B cell responses in SARSCoV-2 naïve and recovered individuals following mRNA vaccination. Sci. Immunol. 6, 1-19 (2021).

18. Jalkanen, P. et al. COVID-19 mRNA vaccine induced antibody responses against three SARS-CoV-2 variants. Nat. Commun. 12, 1-11 (2021).

19. Khoury, D. S. et al. Neutralizing antibody levels are highly predictive of immune protection from symptomatic SARS-CoV-2 infection. Nat. Med. 27, (2021).

20.Lozano-Ojalvo, D. et al. Differential Effects of the Second SARSCoV-2 mRNA Vaccine Dose on T Cell Immunity in Naïve and COVID-19 Recovered Individuals. SSRN Electron. J. 1-9 (2021). doi:https://doi.org/10.1101/2021.03.22.436441

21. Levi, R. et al. One dose of SARS-CoV-2 vaccine exponentially increases antibodies in individuals who have recovered from symptomatic COVID-19. J. Clin. Invest. 131, (2021).

22. Krammer, F. et al. Antibody Responses in Seropositive Persons after a Single Dose of SARS-CoV-2 mRNA Vaccine. N. Engl. J. Med. 384, 1372-1374 (2021).

23. Ebinger, J. E. et al. Antibody responses to the BNT162b2 mRNA vaccine in individuals previously infected with SARS-CoV-2. Nat. Med. 27, 981-984 (2021).

24. Frieman, M. et al. SARS-CoV-2 vaccines for all but a single dose for COVID-19 survivors. EBioMedicine 68, 103401 (2021).

25. Turner, J. S. et al. SARS-CoV-2 mRNA vaccines induce persistent human germinal centre responses. Nature (2021). doi:10.1038/ s41586-021-03738-2

26.Zamora-Ledezma, C.; C., D.F.C.; Medina, E.; Sinche, F.; Santiago Vispo, N.; Dahoumane, S.A.; Alexis, F. Biomedical Science to Tackle the COVID-19 Pandemic: Current Status and Future Perspectives. Molecules 2020, 25, 4620. https://doi.org/10.3390/molecules 25204620 


\section{DE LA CURIOSIDAD ACADÉMIICA A LA INNOVACIÓN TECNOLÓGICA}

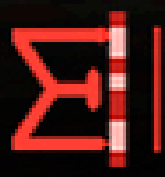

ESCUELA DE CIENCIAS MATEMÁTICAS Y COMPUTACIONALES

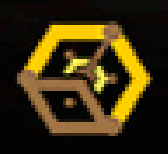

ESCUELA DE

CIENCIAS FISICAS

Y NANOTECNOLOGIA
ESCUELA DE

CIENCIAS QUIMICAS

E INGENIERIA
ESCUELA DE

CIENCIAS DE LA TIERRA,

ENERGIA Y AMBBIENTE

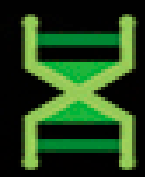

ESCUELA DE

CIENCIAS BIOLÓGICAS

E INGENIERIA 